\title{
DETERMINAÇÃO DA DIFUSIVIDADE E ENERGIA DE ATIVAÇÃO PARA BANANA VERDE E MADURA E ANÁLISE DA CINÉTICA DE SECAGEM PELO PROCESSO FOAM-MAT
}

\author{
L. B. ARAUJO ${ }^{1}$, C. E. M. R. GURGEL ${ }^{2}$ e M. F. D. MEDEIROS ${ }^{1}$ \\ ${ }^{1}$ Universidade Federal do Rio Grande do Norte, Departamento de Engenharia Química \\ ${ }^{2}$ Universidade Federal do Rio Grande do Norte, Programa de Pós-Graduação em Engenharia \\ Química \\ E-mail para contato: laryssaphb@ hotmail.com
}

\begin{abstract}
RESUMO - A técnica de secagem foam-mat consiste na desidratação da espuma obtida através do batimento de líquidos ou pastas com adição de substâncias emulsificantes por meio do contato da superfície da emulsão com ar aquecido. É um processo com baixo custo de operação e alto rendimento que utiliza pequeno tempo de secagem devido à elevada área de contato exposta ao ar aquecido. Estudou-se a cinética de secagem da espuma de banana madura e verde utilizando albumina como agente emulsificante. Aos dados experimentais da razão de umidade em função do tempo foi ajustado o modelo de Page, que se mostrou adequado para representar a cinética de secagem da espuma de ambas as bananas. O coeficiente de difusão efetiva representa a interação de todos os parâmetros que influenciam na transferência de massa. Para obtê-lo, foi aplicada uma solução analítica à segunda lei de Fick, truncada no sétimo termo. Os valores de difusividade encontrados neste trabalho estão dentro da faixa relatada em ensaios com alimentos. A energia de ativação para que ocorra a difusão foi calculada pela relação dos valores de difusividade e a equação de Arrhenius.
\end{abstract}

\section{INTRODUÇÃO}

A banana é uma das frutas mais consumidas no mundo devido a seu alto teor nutricional, baixo custo além de cultivo simples e colheita permanente durante todo o ano. A polpa da banana verde é uma massa sem sabor, com baixo teor de açúcares (RODRÍGUEZ-AMBRIZ et al., 2008), sendo o amido o principal componente, representando de $55 \%$ a $93 \%$ da quantidade de sólidos totais do fruto (EMBRAPA, 2009). Já a banana madura possui polpa macia e doce, com uma quantidade considerável de carboidratos, $23 \%$, que são facilmente assimiláveis, e cerca de 1,1\% de proteínas (USDA, 2011). Entretanto, a fruta apresenta um rápido escurecimento devido à atividade enzimática que é ligada à quantidade de água presente no fruto, diminuindo sua vida de prateleira e limitando sua aplicação.

A secagem é um processo largamente utilizado na indústria de polpas de frutas, pois concentra os componentes da matéria-prima e aumenta o tempo de conservação, diminuindo perdas. A secagem em camada de espuma, ou foam-mat, consiste na desidratação da espuma formada pelo batimento de líquidos ou pastas com adição de substâncias emulsificantes em 
contato com ar quente, resultando em um pó de baixa umidade. É um processo vantajoso devido ao seu baixo custo, alto rendimento.

Neste trabalho, foi utilizada uma camada de $1,8 \mathrm{~cm}$ nas secagens e albumina como agente emulsificante, na concentração de $7,5 \%$ para formação da espuma. Foram realizados três ensaios em temperaturas diferentes para cada tipo de banana $\left(60^{\circ} \mathrm{C}, 70^{\circ} \mathrm{C}\right.$ e $\left.80^{\circ} \mathrm{C}\right)$, e aos dados de secagem foram ajustados o modelo de Page.

O coeficiente de difusividade efetiva é importante para projetar e otimizar processos que envolvam transporte interno de umidade (PRASAD et al., 2010). Ele representa a interação de todos os parâmetros que influenciam a transferência de massa e aqui foi obtido a partir de uma solução analítica da segunda lei de Fick, truncada no sétimo termo. Relacionando os valores da difusividade com a equação de Arrhenius foi possível calcular a energia de ativação necessária para que a difusão ocorra.

\section{METODOLOGIA}

\subsection{Preparação do purê e da espuma}

Foram utilizadas bananas do tipo Pacovan e a metodologia para os dois tipos de banana foi a mesmo. As bananas eram descascadas e cortadas em rodelas, sendo tratadas com metabissulfito de sódio para evitar o escurecimento durante o batimento. A banana ficava imersa na solução por 1 minuto, depois era lavada com água destilada por 30 segundos. As rodelas eram então batidas em um processador doméstico para a formação da pasta de banana. Para a obtenção da espuma, era adicionada albumina previamente pesada na concentração de 7,5\% da massa da pasta e a mistura era batida com batedeira comum em velocidade máxima por 4 minutos.

\subsection{Secagem}

A espuma obtida era distribuída em uma placa de alumínio com $1,8 \mathrm{~cm}$ de espessura e levada a estufa de circulação de ar regulada na temperatura desejada para o experimento. Para cada tipo de banana foi realizado três ensaios, cada um com uma temperatura diferente $\left(60^{\circ} \mathrm{C}\right.$, $70^{\circ} \mathrm{C}$ e $80^{\circ} \mathrm{C}$ ). Durante a secagem, a placa era tirada da estufa e pesada para medir a quantidade de água evaporada naquele intervalo de tempo. Quando o fim da secagem se aproximou esse intervalo foi aumentado para trinta minutos para que uma mudança significativa fosse notada na massa. Quando a massa ficou contestante, a secagem foi interrompida.

\subsection{Aplicação do Modelo de Secagem}

Aos dados da cinética de secagem foram aplicados o modelo de Page, pois este representa a perda de água dos alimentos durante a secagem e é descrito pelas equações 1 e 2 , abaixo. O software utilizado para o ajuste do modelo foi o Statistica 7.0.

$$
\begin{aligned}
& R U=\frac{U_{b s}-U_{e q}}{U_{b s i}-U_{e q}} \\
& R U=e^{-k t^{n}}
\end{aligned}
$$


Onde RU é a razão de umidade, $U_{b s}$, $U_{\text {eq }}$ e $U_{b s i}$ são umidades em base seca, no equilíbrio e em base seca inicial, k é o parâmetro do modelo de Page $\left(\mathrm{min}^{-\mathrm{n}}\right)$, n é o parâmetro adimensional do modelo e té o tempo em minutos.

\subsection{Obtenção da difusividade efetiva}

O coeficiente de difusividade efetiva foi obtido através da solução analítica da segunda lei de Fick (equação 3), truncada no sétimo termo.

$$
\mathrm{RU}=\frac{8}{\pi^{2}} \sum_{\mathrm{n}=0}^{7} \frac{1}{(2 \mathrm{n}+1)^{2}} \operatorname{Exp}\left(-\left(\frac{(2 \mathrm{n}+1) \pi}{\mathrm{a}}\right)^{2} \mathrm{D}_{\mathrm{ef}} \mathrm{t}\right)
$$

Onde RU é a razão de umidade, $\mathrm{D}_{\text {ef }}$ é o coeficiente de difusividade efetiva $\left(\mathrm{m}^{2} / \mathrm{s}\right)$, a é a espessura da placa em metros, $n$ é o número de termos e t é o tempo em segundos.

\subsection{Energia de ativação}

Os valores da difusividade efetiva podem ser relacionados com a elevação da temperatura do ar de secagem por meio da equação de Arrhenius (equação 4). Para tal, foi utilizado o Microsoft Office Excel.

$$
\mathrm{D}_{\mathrm{ef}}=\mathrm{D}_{\mathrm{o}} \operatorname{Exp}\left(\frac{-\mathrm{E}}{\mathrm{RT}}\right)
$$

Onde $\mathrm{D}_{\mathrm{o}}$ é o fator pré-exponencial, E é a energia de ativação, $\mathrm{R}$ é a constante dos gases $\left(8,314 \mathrm{~J} \mathrm{~K}^{-1} \mathrm{~mol}^{-1}\right)$ e T é a temperatura em Kelvin.

\section{RESULTADOS E DISCUSSÕES}

Antes do início da secagem, foi feita uma caracterização das espumas a serem secadas quanto a sua umidade e teor de sólidos solúveis ( ${ }^{\circ}$ Brix). Os valores obtidos experimentalmente são expostos na Tabela 1, para ambos os tipos de banana.

Tabela 1 - Caracterização das espumas de banana madura e verde

\begin{tabular}{|c|c|c|c|c|c|c|}
\hline & \multicolumn{3}{|c|}{ Banana Madura } & \multicolumn{3}{c|}{ Banana Verde } \\
\hline \hline $\begin{array}{c}\text { Temperatura } \\
(\mathrm{K})\end{array}$ & Umidade (\%) & $\begin{array}{c}\text { Desvio } \\
\text { Padrão }\end{array}$ & $\begin{array}{c}\text { Sólidos } \\
\text { Solúveis } \\
\left({ }^{\circ} \text { Brix) }\right.\end{array}$ & Umidade (\%) & $\begin{array}{c}\text { Desvio } \\
\text { Padrão }\end{array}$ & $\begin{array}{c}\text { Sólidos } \\
\text { Solúveis } \\
\left({ }^{\circ} \text { Brix) }\right.\end{array}$ \\
\hline \hline 333 & 64,643 & 0,380 & 25,037 & 65,810 & 0,453 & 6,883 \\
\hline 343 & 66,323 & 0,206 & 23,527 & 66,808 & 0,442 & 6,990 \\
\hline 353 & 64,165 & 0,049 & 23,000 & 67,955 & 0,003 & 7,040 \\
\hline
\end{tabular}

Nota-se que o teor de sólidos solúveis na banana madura é bem mais elevado que na banana verde, evidenciando a alta concentração de açúcares na banana madura e a presença de amida na banana verde. 
As curvas de secagem com os dados experimentais e preditas pelo modelo de Page são apresentadas na Figura 1, abaixo. A banana madura está indicada por A, e a verde, por B.

Figura 1 - Curvas de secagem da banana madura (A) e banana verde (B), ajustadas ao modelo de Page

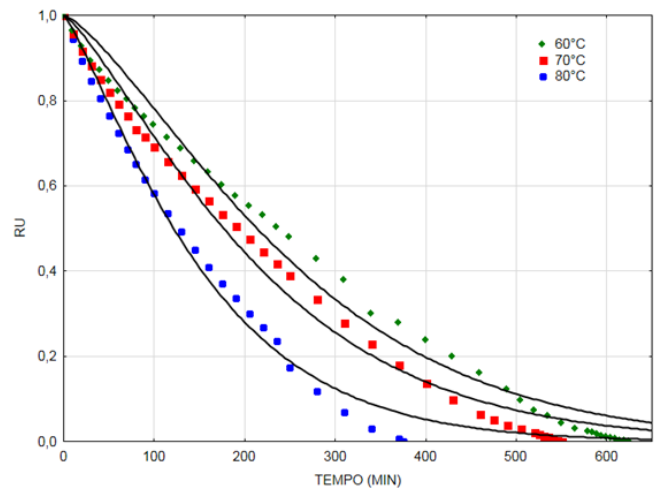

A

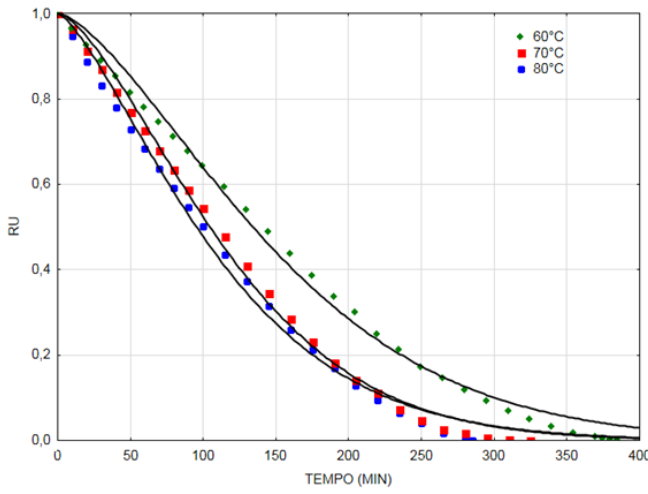

B

Observa-se que, para ambos os tipos de banana, a secagem apresentou o comportamento clássico descrito na literatura. No início, a queda de umidade é alta, diminuindo linearmente com o tempo, o que caracteriza o período de taxa constante. Com o passar do tempo a diminuição da umidade é menos acentuada caracterizando o período de taxa decrescente até atingir um valor constante, quando as condições de equilíbrio são atingidas. Nas curvas também é possível constatar que a secagem da banana madura, embora mais demorada que a da verde para todas as temperaturas estudadas, apresenta o período de constante bastante acentuado.

Na Tabela 2 exibe-se os parâmetros do modelo de Page ajustados aos dados da cinética de secagem das espumas de banana madura e verde.

Tabela 2 - Parâmetros do modelo de Page da banana verde e madura

\begin{tabular}{|c|c|c|c|c|}
\hline $\begin{array}{c}\text { Tipo de } \\
\text { banana }\end{array}$ & Temperatura $(\mathrm{K})$ & $\mathrm{K}\left(\mathrm{min}^{-\mathrm{n}}\right)$ & $\mathrm{N}$ & $\mathrm{R}^{2}$ \\
\hline \hline \multirow{3}{*}{$\begin{array}{c}\text { Banana } \\
\text { Verde }\end{array}$} & 333 & 0,00047 & 1,488 & 0,994 \\
\cline { 2 - 5 } & 343 & 0,00060 & 1,515 & 0,996 \\
\hline & 353 & 0,00127 & 1,383 & 0,994 \\
\hline \multirow{2}{*}{$\begin{array}{c}\text { Banana } \\
\text { Madura }\end{array}$} & 333 & 0,00049 & 1,353 & 0,985 \\
\cline { 2 - 5 } & 343 & 0,00095 & 1,275 & 0,987 \\
\cline { 2 - 5 } & 353 & 0,00002 & 1,218 & 0,990 \\
\hline
\end{tabular}

Observando os valores do coeficiente de correlação $\mathrm{R}^{2}$, superiores a 0,985 em todos os experimentos das bananas verde e da madura, conclui-se que o modelo de Page apresenta um excelente ajuste aos dados das cinéticas de secagem das espumas de banana

Os valores de $\mathrm{K}$ indicam uma taxa específica média de secagem. Como esperado, quanto maior a temperatura, maior o valor da taxa de secagem, comportamento observado nas curvas 
experimentais apresentadas na Figura 1. Também se nota que os valores de $\mathrm{K}$ da banana madura foram maiores que os da banana verde. Isso pode ser explicado pela relação do valor de $\mathrm{K}$ com os dois períodos de secagem, e a predominância do período de taxa constante na secagem da espuma da banana madura.

Utilizando a solução analítica para a segunda lei de Fick (equação 3), foram determinados os valores da difusividade efetiva para os dois tipos de banana, em cada uma das temperaturas e os resultados são organizados na Tabela 3 , abaixo.

Tabela 3 - Valores da difusividade efetiva $x 10^{-08}\left(\mathrm{~m}^{2} . \mathrm{s}^{-1}\right)$

\begin{tabular}{|c|c|c|}
\hline Temperatura $(\mathrm{K})$ & $\operatorname{Def}_{\mathrm{BM}}$ & $\operatorname{Def}_{\mathrm{BV}}$ \\
\hline \hline 333 & 0,715 & 2,589 \\
\hline 343 & 0,871 & 2,752 \\
\hline 353 & 1,206 & 3,004 \\
\hline
\end{tabular}

Observa-se que todos os valores foram na casa de $10^{-08}$. Esses valores estão de acordo com a faixa relatada para ensaios com alimentos, que varia entre $10^{-12}$ e $10^{-08}\left(\mathrm{~m}^{2} \cdot \mathrm{s}^{-1}\right)$ (MCMINN \& MAGEE, 1999).

Nota-se claramente que o valor da difusividade efetiva aumenta com a temperatura e é menor para a banana madura em todas as condições. Isso pode ser explicado pois o modelo da lei de Fick é aplicado apenas ao período de taxa decrescente da secagem, onde a banana madura já perdeu boa parte da sua umidade, refletindo em uma baixa difusividade.

Para determinar a energia de ativação, que segundo Kashaninejad et al. (2007) indica a barreira a ser vencida para que ocorra a difusão da água através no produto, foi aplicada aos valores de difusividade em função do inverso da temperatura a relação de Arrhenius, representada 1 graficamente representada na Figura 2.

Figura 2 - Gráfico da relação da difusividade com a temperatura

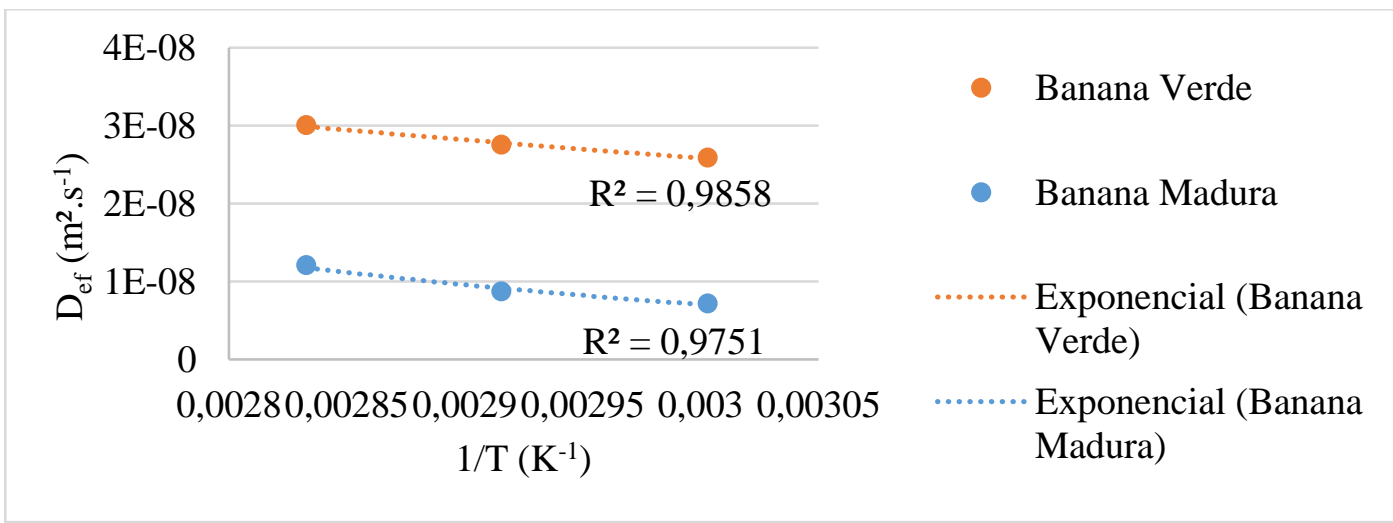

Nas condições abordadas neste trabalho, a energia de ativação para a difusão na banana verde é de 7,246 kJ/mol, e para banana madura é de $25,499 \mathrm{~kJ} / \mathrm{mol}$. Observa-se que quanto maior a difusividade, menor a energia de ativação necessária para que ela ocorra. 
Vários outros autores estudaram a energia de ativação para a difusão em alimentos, como Resende et al. (2007), que encontraram o valor de $6,748 \mathrm{~kJ} / \mathrm{mol}$ para o feijão, e Furtado et al (2010) que encontraram valores entre 36 e $40,81 \mathrm{~kJ} / \mathrm{mol}$ para a polpa de ceriguela para secagens entre 60 e $80^{\circ} \mathrm{C}$. Zogzas et al. (1996) apresentam a faixa entre 12,7 e $110 \mathrm{~kJ} / \mathrm{mol}$ para produtos agrícolas. As energias de ativação encontradas estão em concordância com as de outros alimentos encontradas na literatura. Os valores relativamente baixos encontrados neste estudo indicam que a banana verde e madura tem maior facilidade para absorver água (Resende et al., 2007).

\section{CONCLUSÃO}

De maneira geral, o aumento da temperatura do ar de secagem influencia tanto na taxa de secagem quanto na difusividade efetiva. O modelo de Page ajustado aos dados experimentais apresentou ótimos coeficientes de correlação, todos acima de $98 \%$, o que indica que o modelo descreve bem a secagem. Com o aumento da temperatura ao coeficiente K que representa uma taxa específica média de secagem também aumenta, indicando uma secagem mais rápida. Vale ressaltar que esse coeficiente se relaciona com os dois períodos da secagem. A predominância do período de taxa constante na secagem da banana madura, onde há uma perda acentuada de umidade, faz com que os valores de $\mathrm{K}$ sejam maiores que para a banana verde. Os coeficientes de difusividade obtidos são da ordem e grandeza de $10^{-09}$ e $10^{-08}$, dentro da faixa esperada para ensaios com alimentos e aumenta com a temperatura. A energia de ativação apresentada pela banana verde foi 7,246 kJ/mol, e 25,499 kJ/mol pela banana madura, valores condizentes com os encontrados para outros alimentos na literatura.

\section{REFERÊNCIAS}

FURTADO, G de. F.; SILVA, F. S. da; PORTO, A. G.; SANTOS, P. dos. Secagem de polpa de ceriguela pelo método de camada de espuma. Revista Brasileira de Produtos Agroindustriais, Campina Grande, v. 12, n. 1, p.9-14, 2010.

KASHANINEJAD, M.; MORTAZAVI, A.; SAFEKORDI, A.; TABIL, L. G. Thin-layer drying characteristics and modeling of pistachio nuts. Journal of Food Engineering, v.78, p.98-108, 2007.

MCMINN, W. A. M.; \& MAGEE, T. R. A. (1999). Principles, methods and applications of the convective drying of food stuffs. Trans IChemE, 77, 175-193.

PRASAD, K., VAIRAGAR, P. R., BERA, M. B. Temperature dependente hydration kinetics of Cicer arietinum splits. Food Research International, v.43, n.2, p.483-488, 2010.

RAJKUMAR, P. et al. Drying characteristics of foamedalphonso mango pulp in a continuous type foam mat dryer. J. Food Eng., v. 79, p.1452-1459, 2007

RESENDE, O.; CORRÊA, P. C.; JARÉN, C.; MOURE, A. J. Bean moisture diffusivity and drying kinetics: a comparison of the liquid diffusion model when taking into account and neglecting grain shrinkage. Spanish Journal of Agricultural Research, v.5, p.51-58, 2007.

Zogzas, N. P.; Maroulis, Z. B.; Marinos-Kouris, D. Moisture diffusivity data compilation in foodstuffs. Drying Technology, v.14, p.2225-2253, 1996. 